\title{
Objective Evaluation for Wrinkle Appearance of Fabrics by Image Processing and Slit Beam Projecting Technique
}

\author{
Jian HAN*, Minzhuang YANG*, and MATSUDAIRA Mitsuo ** \\ * Graduate School of Natural Science, Kanazawa University, Kanazawa City 920-1192,Japan \\ **Faculty of Education, Kanazawa University, Kakuma-machi, Kanazawa City 920-1192,Japan
}

Received 14 July, 2002; Accepted for Publication 6 December, 2002

\begin{abstract}
The slit beam projecting technique combined with the image processing is used, and three-dimensional image of the wrinkle of fabrics is obtained by the method, in which there is no influence on the results from the colors and the patterns of fabrics, and the fabric wrinkles can be evaluated and analyzed objectively. The parameters, such as standard deviation of height values of surface profiles, increasing ratio of surface area, one of surface length, and fractal dimension of surface, are defined and suggested to evaluate and characterize the fabric wrinkles. There are logarithm relationships between the parameters and the wrinkle grades of standard replicas, and their correlations are high, too. There is a good agreement between the subjective appraisal and the objective evaluation results for the fabrics.
\end{abstract}

Key words: Wrinkle, Image processing, Slit beam projecting, Subjective appraisal, Objective evaluation

\section{Introduction}

Aesthetic appearance is one of the most important criteria used by the customs and manufacturers in judging the total wear performance of clothing. The outward appearance of a garment and its easy care properties worsen due to wrinkling during wearing or after repeated home laundering. Traditionally, subjective evaluation ${ }^{[1-3]}$ was still a main method to evaluate the wrinkle appearance of fabrics, but the results using the method were affected by visual difference of human and other factors. And furthermore, the method sometimes leaded to inconsistent results. Recently, objectively evaluating methods ${ }^{[4-11]}$ had widely been studied. One of them used the image processing to measure it. Its principle made use of the change of gray level of the reflection light on the fabric surface with the influence from wrinkle. However, it would cause some limitation and inappropriateness. The analyzed results mainly depended on the light sources, the colors or the prints of fabrics, and the reflection property of surface. And, it should meet in difficulty to evaluate some fabrics having dark colors or prints. The other utilized an alligned grid projecting technique to radiate grid lines on the fabric surface tested. After the image was applied through the filtering, geometric correction and scanning procedure, the profiles for locating grid line position and height values of surface were obtained. The method could be used to evaluate objectively the wrinkle properties of some fabrics of colored or patterned design. But, it still had some problems that need to be resolved. Since there were about 30 grid lines radiated on the fabric, it increased the illumination on fabric surface and led that the difference of gray level between grid line and fabric was lowered. Such, the method could not be used to measure the fabrics having sharply contrasting colors or prints. Besides, because the captured image was a trapezium, a part of grid line farther away the camera did not come into focus. The measured area was limited and the produced points were also limited, owing to interval scanning. To overcome the aforementioned defects and to improve the disadvantage, the slit beam projecting technique combining with image processing is developed and used as a 3-D measurement system to evaluate the wrinkle appearance objectively and wrinkle grades of fabrics in the study. The measuring results and the relationship between subjective appraisal and 
objective measurement are also investigated.

\section{Experimental Equipment and Methods}

\subsection{3-D Measurement of Fabric Surface} Wrinkle

The slit beam projecting technique is a versatile tool for grabbing high-precision $3 \mathrm{D}$ range images of an objective surface and measuring the surface profile and warpage for various engineering materials and structures with displacement resolution. The principal elements in the system include a light projector, a CCD camera, a moving platform, a monitor and a personal computer, in which a capture board is installed. As shown in Figure 1, a profile line, which is $0.6 \mathrm{~mm}$ in width, is radiated onto the surface of a sample to be tested in a dark room and an image is captured with the CCD camera. The incidence angle and the angle between the CCD camera and Z-direction are $25^{\circ}$ and $55^{\circ}$, respectively. Before the sample is deformed, the projected line is a standard slit one. When the sample is wrinkled or curved, the projected line will be distorted. By comparing the distorted line with the original reference plane, the surface height of the sample can be detected. The height ' $Z_{i}$ ' of a point on sample can be deduced by the shift ' $\mathrm{S}_{\mathrm{i}}$ ' in the camera image. By means of height calibration, the value $\left(Z_{i}\right)$ of surface height can be obtained. Using a spatial resolution of $80 \times 361$ pixels, corresponding to approximately $25 \times 110$ $\mathrm{mm}$ in actual area, the reflected images are captured at intervals of $2 \mathrm{~mm}$ when the platform and sample moves along $\mathrm{X}$ direction. There are 41 images for every specimen, and they are made up a total tested area $(80 \times 110 \mathrm{~mm})$ in the center of sample. The images are processed on the computer in order to determine the profile information. The system process is shown in Figure 2. An original image (a) is firstly captured. Because there is a clear difference in gray level between the dim background and the bright profile line on specimen in the image for any replica or fabric having colors or prints, including those with sharp color contrasts, the satisfactory binary image can be easily and simply gotten. Secondly, the image is changed into binary one (b), and then the curve is thinned to obtain the thinning image(c). Actually, the thinning image (d) with 41 curves is composed during 41 curves in every sample are simultaneously thinned. Finally, after shape data are filtered, computed and analyzed, high-precision $3 \mathrm{D}$ range images are obtained and wrinkle appearances of surface and wrinkle grades of fabrics are evaluated in the system. In order to analyze and compare the measurement results with the subjective appraisals, 10 fabrics are used for the experiment with the various colors or prints. According to method of AATCC 128 , the rectangular specimens $150 \times 280 \mathrm{~mm}$ in the warp direction are cut, and then, are kept in the standard atmosphere at $65 \%$ of $\mathrm{RH}$ and $20^{\circ} \mathrm{C}$ for 24

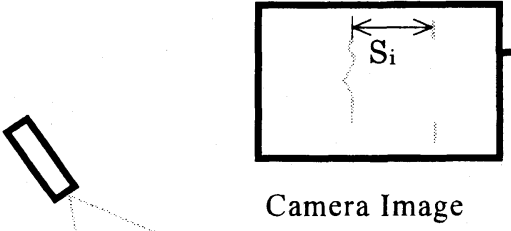

Slit Projecting Beam

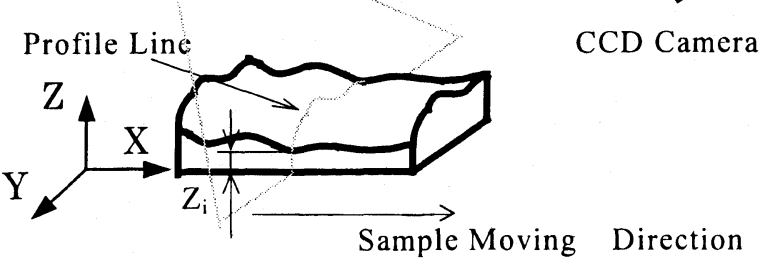

Fig.1 Schematic diagram of 3D measurement system
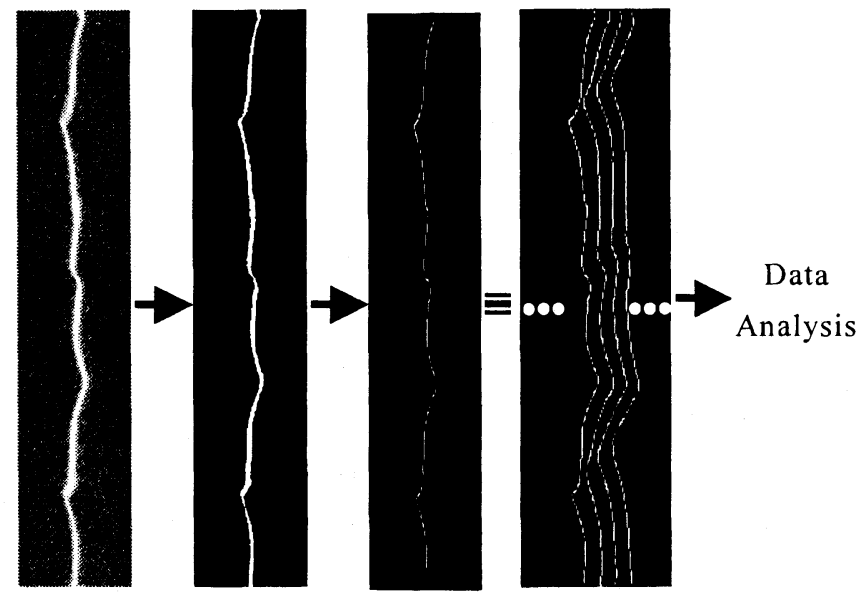

(a) (b)

(c)

(d)

(e)

Fig. 2 Process of the experiment

(a)Original image (b) Binary image (c), (d) Thinning image (e) Filtering and Data analysis 
hours in advance. The $3.5 \mathrm{~kg}$ weights are left in situs for 20 minutes, and then this period of time has elapsed. After 24 hours recovery periods, wrinkle recovery is measured and assessed. Subjective appraisal, of which wrinkle grade $\left(\mathrm{WG}_{\mathrm{sb}}\right)$ is the average of results, is assessed by four specialists against the set of standard replica and objective one is measured by this system.

\subsection{FFT Filtering}

Because of the illumination reflection and the surface nuance of replica or fabrics, there exists slight un-uniformity for the profile line in width in the image. The noise is produced in processing of binary and thinning images. All signals are filtered using Fast Fourier Translation (FFT) Filter, and the noises with high frequency signals have been wiped off without adversely affecting the signals.

\section{Characterization of Wrinkling}

The wrinkle properties can be judged by means of analyzing the height of surface profiles, and they are shown by the parameters $Z_{\mathrm{SD}}, \eta_{\mathrm{A}}, \eta_{\mathrm{Lx}}, \eta_{\mathrm{Ly}}$, and FD, which represents the standard deviation of height of curves, the increasing rate of surface area, the length increasing rate of surface curves in $X$ and in $\mathrm{Y}$ direction, and Fractal dimension of surface, respectively.

\subsection{Standard deviation of height values of surface profiles}

In this study, a typical profile line of surface is shown in Figure 3. If a height function of surface profiles is given as $Z=f(x, y)$, the standard deviation of height values $\left(Z_{\mathrm{SD}}\right)$ can be expressed by

$$
Z_{S D}=\sqrt{\frac{\sum \sum\left(Z_{(i, j)}-\bar{Z}\right)^{2}}{m \times n}}
$$

where $\mathrm{Z}_{(\mathrm{i}, \mathrm{j})}$ is the height value of point $\mathrm{A}(\mathrm{i}, \mathrm{j}), \bar{Z}$ the mean of data points, $m=41$, the number of data points in $X$ direction, and $n=361$, the one in $Y$ direction, separately. Height values of surface profiles can be used to describe the wrinkle appearance. A smooth fabric is expected to exhibit a uniform appearance, resulting in a low standard deviation. Distribution of height values is obviously varied with increasing of wrinkle, although the mean of height values in the measured area range is slightly changed. It is clear that the larger the standard deviation of height values is, the more wrinkled that fabric becomes.

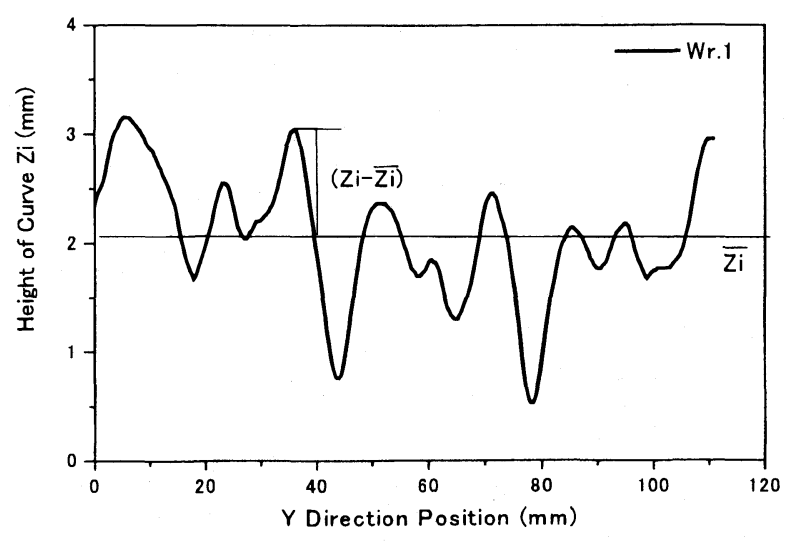

Fig. 3 A typical profile line of surface

\subsection{Increasing rate of surface area $\eta_{\mathrm{A}}$}

A surface area chiefly depends on the characteristics of a surface, and reflects the wrinkle grade. To calculate surface area, four adjacent points (A, B, C and D) sharing one quadrilateral are first selected. The sum surface area is calculated by summing individual quadrilateral. The method calculating the surface area and the length is addressed in detail by us ${ }^{[10]}$. The increasing rate of surface area $\eta_{\mathrm{A}}$ is defined as follows:

$$
\eta_{A}=\frac{\sum S_{A^{\prime} B^{\prime} C^{\prime} D^{\prime}}-\sum S_{A B C D}}{\sum S_{A B C D}} \times 100 \%
$$

where $\Sigma S_{A^{\prime} B^{\prime} C^{\prime} D^{\prime}}$ is total surface area of the vertexes (A', B', C' and $\mathrm{D}^{\prime}$ ), $\Sigma \mathrm{S}_{\mathrm{ABCD}}$ is total orthogonal projection area in $\mathrm{X}-\mathrm{Y}$ plane, that is, it is the plane area which any wrinkle is not in. Obviously, the larger the increasing rate of surface area $\eta_{A}$ is, the higher wrinkle grade that fabric appears to be.

\subsection{Length increasing rate of surface in $X, Y$ direction $\eta_{\mathrm{Lx}}, \eta_{\mathrm{Ly}}$}

According to the characteristics of fabric wrinkle after induced wrinkling with Wrinkle Recover Tester of AATCC, their length increasing rate of surface profiles in X-direction and Y-direction will be different. $\eta_{\text {Lx }}$ and $\eta_{\text {Ly }}$ are defined as follows: 


$$
\begin{aligned}
& \eta_{l . x}=\frac{\sum A^{\prime} B^{\prime}-\sum A B}{\sum A B} \times 100 \% \\
& \eta_{I, y}=\frac{\sum A^{\prime} C^{\prime}-\sum A C}{\sum A C} \times 100 \%
\end{aligned}
$$

\subsection{Fractal dimension of surface profiles (FD)}

Fractal is proposed by D.Sci. Benoit B. Mandelbrot, and is a geometry based on the self-similarity for the purpose to analyze and to describe the complex shape and the pattern. Fractal dimension quantifies the complexity of the shape characteristics and the coarseness of surface. Recent years, this method has been becoming to be more and more widely used in the field to evaluate the complexity of fiber crimp and fabric wrinkles [12,13]. Usually, FD of a surface curve can be expressed as follows:

$F D=-\frac{\log N(r)}{\log r}$

where $r$ is the unit scale length and $N(r)$ the numbers segmented by unit scale. In the case of $F D=2$, the surface implies a perfectly smooth plane, and when FD approaches 3 , it does the rugged and complex surface.

There are several methods to estimate the Fractal dimension, and wavelet method is used to do it in our work. After a surface is cut through the cross section in $\mathrm{X}$ and $\mathrm{Y}$ direction, FD of surface can be estimated using the fractal processing program.

\section{Results and Discussions}

The slit beam projecting technique combining with image processing is developed and used as a 3-D measurement system to evaluate the wrinkle appearance objectively.

Figure 4 shows 3 -D surface shape of AATCC standard replicas with $80 \times 80 \mathrm{~mm}$ in the center. Because there exists an uneven and a somewhat nonlinear behavior of wrinkle grade in the replicas, disparity and difference between wrinkle grade 1 and 2 are very significant, but ones between wrinkle grade 4 and 5 are too small. Figure 5 is a contour plot of wrinkle grade 1, 3 and 5 showing height of surface and presents the obvious differences among them. The range of height value in wrinkle grade 1 is distributed from 0 to $3.5 \mathrm{~mm}$, its contour map is very intricate. On the contrary, the range in wrinkle grade 5 is done from 0 to $1 \mathrm{~mm}$, its map is also the simplest.

Figure 6 indicates relationship between standard deviation of height values of surface profiles $Z_{S D}$ and

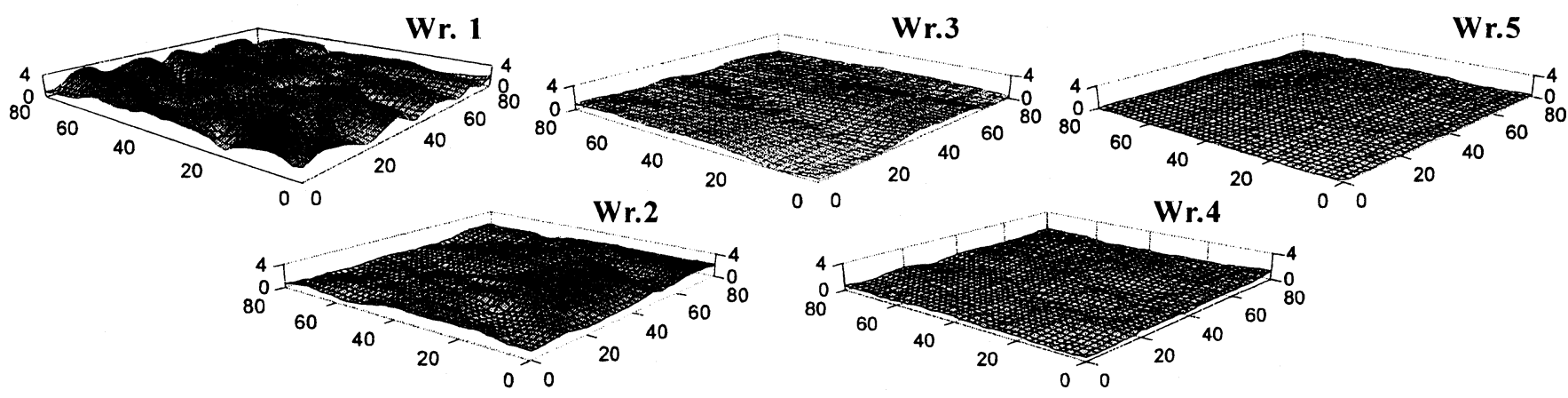

Fig.4 3-D surface shape of replicas

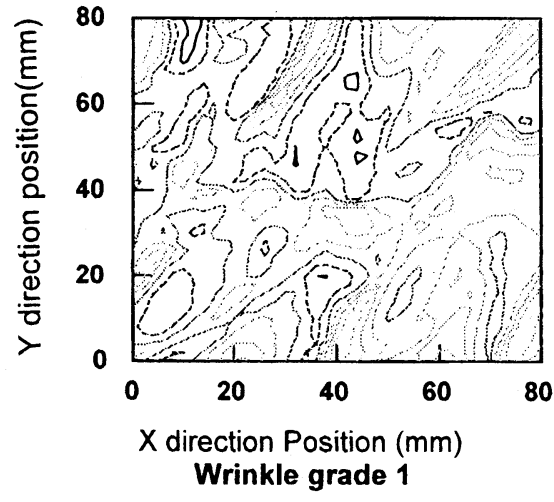

Wrinkle grade 1
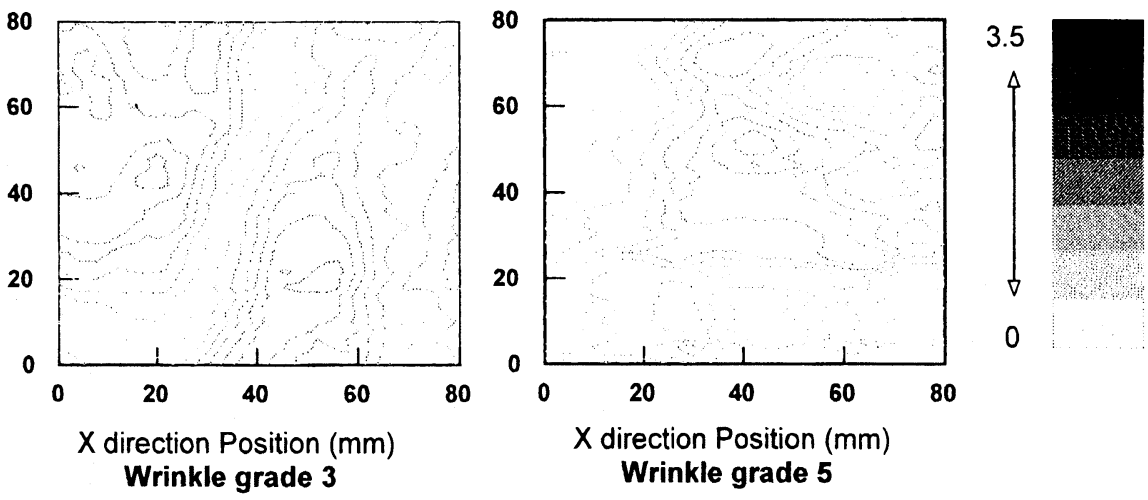

Fig. 5 Contour plot showing height of surface 


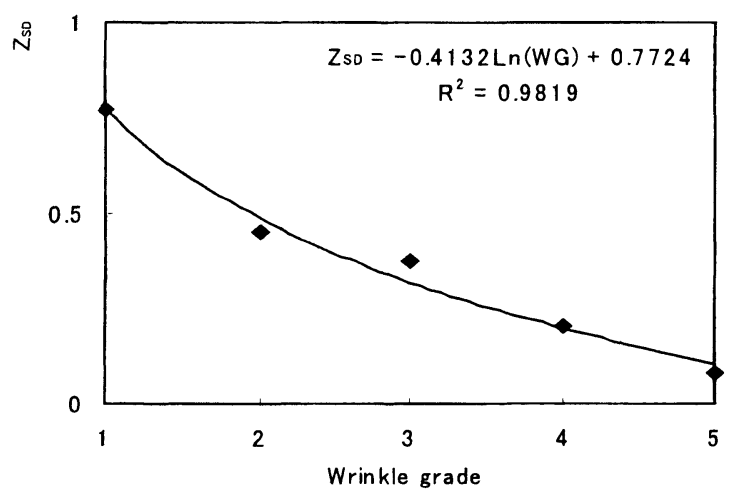

Fig. $6 Z_{S D}$ and wrinkle grade

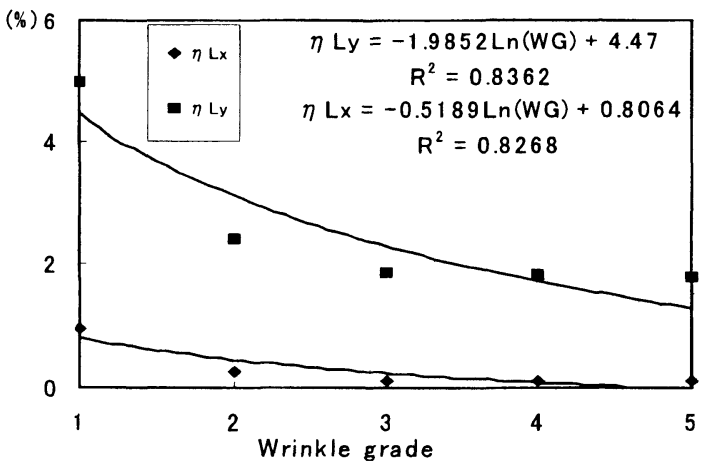

Fig. $8 \eta_{\mathrm{Lx}}, \eta_{\mathrm{Ly}}$ and wrinkle grade

wrinkle grades of replicas. Note, the larger $Z_{S D}$ is, the higher the wrinkle degree, and there is a higher correlation relationship between $Z_{S D}$ and wrinkle grade at $1 \%$ significant level.

The increasing ratio of surface area $\eta_{\mathrm{A}}$ with wrinkling is shown in Figure 7. As shown in Figure 8 , when the wrinkle degree is increased, $\eta_{L x}$ and $\eta_{\text {Ly }}$ also increase. There are good logarithmic functions between $\eta_{\mathrm{A}}, \eta_{\mathrm{L}}$ and wrinkle grade, which have high correlation relationship in every regression curve at $5 \%$ significant level.

In Figure 9, the fractal dimension, one of the parameters for the wrinkle of surface, is plotted to show the trend with change of wrinkle grade, and there is a higher correlation relationship at $1 \%$ significant level.

Among these parameters, $Z_{\mathrm{SD}}, \mathrm{FD}$ and $\eta_{\mathrm{A}}$ show larger differences, while $\eta_{\text {Lx }}, \eta_{\text {Ly }}$ does only subtle ones with the changing of wrinkle grades. So, there are some difficulties to evaluate and measure the wrinkle properties from grade 3 to 5 by using the parameters $\eta_{\text {Lx }}$, and $\eta_{\text {Ly. In }}$ following analysis, we mainly use the parameter $Z_{S D}, \quad \eta_{A}$ and $F D$, and the wrinkle grade of objective evaluation $\left(W G_{b}\right)$ is the mean of wrinkle grades evaluated with three parameters.

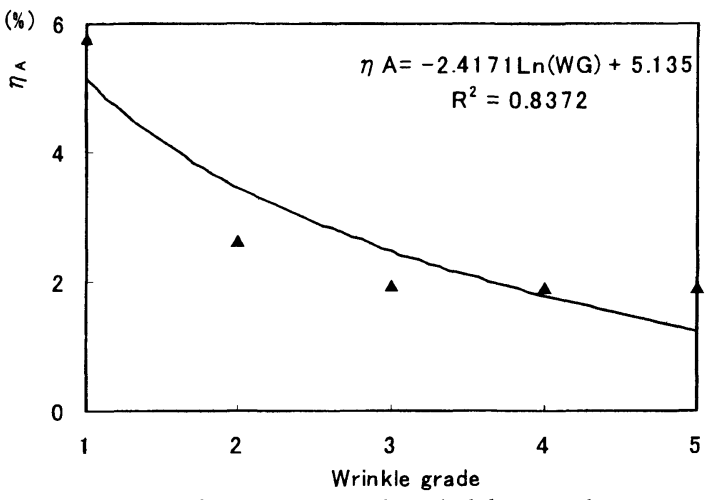

Fig.7 $\eta_{\mathrm{A}}$ and wrinkle grade

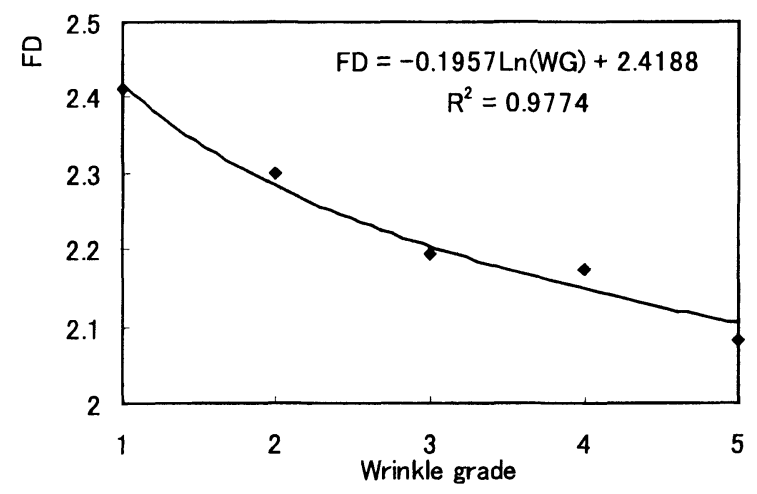

Fig.9 FD and wrinkle grade

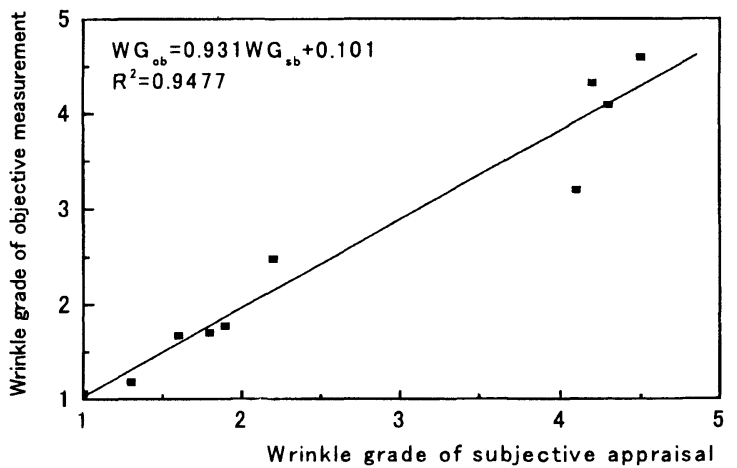

Fig. 10 Results of subjective appraisal and objective measurement

The Table 1 illustrated the results between the subjective appraisal and the objective evaluation for 10 fabrics having the different materials, weave structures, colors or patterns. As shown in Figure 10, there is a high correlation relationship between the wrinkle grades of the subjective appraisal $\left(\mathrm{WG}_{\mathrm{sb}}\right)$ and the ones of the objective evaluation $\left(W G_{o b}\right)$. However, we also find that there are some differences between $\mathrm{W}_{\mathrm{SD}}$ and $\mathrm{W}_{\mathrm{FD}}$ in measuring some fabrics having higher or lower wrinkle appearances, for example linen or polyester fabrics. The reason is that the surface shape of wrinkled linen fabric mainly contains higher 
Table 1 Samples of fabrics and their wrinkle grade appraisals

\begin{tabular}{clllllllll}
\hline Sample & Color & Material & Structure & Weave & WG $_{\text {Sub }}$ & WSD $_{\text {S }}$ & W $_{A}$ & W $_{\text {FD }}$ & WG $_{\text {Ob }}$ \\
\hline 1 & printed & acrylic & filament & plain & 4.1 & 3.88 & 3.41 & 2.31 & 3.20 \\
2 & ivory & cotton & yarn & plain & 1.9 & 1.43 & 2.26 & 1.61 & 1.77 \\
3 & white & cotton & yarn & plain & 1.8 & 1.67 & 1.85 & 1.59 & 1.70 \\
4 & coral & linen & yarn & plain & 1.0 & 0.95 & 0.79 & 1.43 & 1.06 \\
5 & dim gray & polyester & filament & plain & 4.3 & 5.24 & 3.77 & 3.26 & 4.09 \\
6 & olive & polyester & filament & plain & 4.2 & 4.96 & 4.26 & 3.73 & 4.32 \\
7 & ivory & rayon staple & yarn & plain & 1.6 & 2.12 & 1.61 & 1.27 & 1.67 \\
8 & white & rayon & filament & 3-twill & 1.3 & 1.35 & 1.17 & 1.01 & 1.18 \\
9 & ivory & silk & filament & 5-satin & 2.2 & 3.18 & 2.77 & 1.49 & 2.48 \\
10 & black & wool & yarn & plain & 4.5 & 4.70 & 4.23 & 4.84 & 4.59 \\
\hline
\end{tabular}

and larger wrinkles and rarely does lower and smaller ones. It causes a relatively high wrinkle degree using $\mathrm{W}_{\mathrm{SD}}$ and a relatively low one using $\mathrm{W}_{\mathrm{FD}}$. On the contrary, the surface shape of wrinkled polyester is chiefly consisted of lower and smaller wrinkle and does not include larger one. An opposite trend is obtained when it is evaluated with $\mathrm{W}_{\mathrm{SD}}$ or $\mathrm{W}_{\mathrm{FD}}$. $\mathrm{W}_{\mathrm{SD}}$ mainly depends on the height changes and implies the deviation degree from their mean value. Besides the height change, $\mathrm{W}_{\mathrm{FD}}$ also expresses the complexity of changing and the coarseness of surface. However, there is a good agreement between the subjective appraisal $\mathrm{WG}_{\mathrm{sb}}$ and their mean wrinkle grade $W G_{o b}$. In addition, when the print fabric is evaluated, its wrinkle range of subjective appraisal is slight lower than that of objective evaluation. This is because the prints and the patterns affect the visual sensation and it causes that wrinkle degree of subjective appraisal is lowed.

\section{Conclusions}

Projected beam technique, in conjunction with advanced image processing, provides an effective way to measure and evaluate the wrinkle properties of standard replicas and fabrics. The method not only avoids differences affected by vision in subjective appraisals, but also overcomes the defects in evaluating the fabrics having colors and prints with image system. Following conclusions are obtained:

(1) All parameters with wrinkling fall on good logarithmic functions, which have high correlation coefficients.

(2) All parameters used can quantify the wrinkle of replicas and fabrics, and the measurement results are not affected from the colors or the prints of fabrics, including those with sharp color contrasts.

(3) There is good agreement between the subjective appraisal and the objective evaluation results for the fabrics.

\section{References}

[1] C. O. Bostwick; Tefo, No. 3(1961)

[2] Morooka H.,Niwa M.; Text. Mach. Soc. Japan, 17, 176(1976)

[3] Morooka H.,Niwa M.; Text. Mach. Soc. Japan, 18, 27(1977)

[4] Y.J. Na and B. Pourdeyhim; Text. Res. J., 65, 149(1995)

[5] B.Xu and J.A.Reed; J. Text. Inst.,86, 129(1995)

[6] E.H.Kim; Text. Res. J., 69, 860(1999)

[7] T.J.Kang, D.H.Cho, and H.S.Whang; Text. Res. J., 69, 261(1999)

[8] Nishimatsu T.,Ohta K.; Sen'i Gakkaishi, Japan, 56, 348(2000)

[9] Mori T., Wakida T.,and Endou Y.; Sen'i Gakkaishi, Japan, 55, 424(1999)

[10]M.Matsudaira,J.Han,M.Yang; J. Text. Eng. Jpn., 48, No.1, 11(2001)

[11]N.Ota, A.Shibuya; Jpn.Res. Assn.Text. EndUses, 43,284(2002)

[12] T.J.Kang, J.Y.Lee; Text. Res.J.,69,527(1999)

[13] T.J.Kang, J.Y.Lee; Text. Res.J.,70,469(2000) 